\title{
Planejamento agrícola e implantação de sistema de cultivo de cana-de-açúcar com auxílio de técnicas geoestatísticas ${ }^{1}$
}

\author{
Milton C. C. Campos ${ }^{2}$, José Marques Júnior ${ }^{2}$, Gener T. Pereira ${ }^{2}$, Zigomar M. de Souza ${ }^{3}$ \& Rafael Montanari²
}

\begin{abstract}
RESUMO
A posição de importância ocupada pela cana-de-açúcar (Saccharum officinarum) no agronegócio brasileiro justifica o uso de técnicas que propiciem a implantação de um sistema avançado de cultivo, a partir do conhecimento da variabilidade espacial dos atributos do solo. O objetivo deste trabalho foi realizar o planejamento agrícola e a implantação de um sistema de cultivo da gramínea, com o auxílio de técnicas geoestatísticas, na região de Pereira Barreto, SP. Para o mapeamento de uma área de 505 ha utilizando-se equipamento de sistema de posicionamento global (GPS) elaborou-se, em seguida, o Modelo Digital de Elevação (MDE); depois coletaram-se amostras de solo na profundidade de 0-0,25 m, a cada 7 ha, para caracterização química e granulométrica. Os atributos físicos e químicos do solo foram analisados por meio de estatística descritiva e geoestatística. A distribuição espacial dos atributos granulométricos e químicos do solo permitiu alocação das variedades em estudo de acordo com a fertilidade do solo e teor de argila. O mapa de krigagem dos atributos granulométricos e químicos do solo forneceu informações para o estabelecimento dos ambientes de produção, com manejo do solo e de culturas diferenciado. O uso de técnicas geoestatísticas aplicadas na análise dos atributos dos solos permitiu realizar com precisão o planejamento do cultivo de cana-de-açúcar, a partir da separação de ambientes, adequação de práticas de adubação e alocação de variedades adaptadas às restrições impostas pelos atributos dos solos.
\end{abstract}

Palavras-chave: atributos do solo, zonas de manejo específico, krigagem

\section{Agricultural planning and implementation of cultivation systems for sugarcane using geostatistics techniques}

\begin{abstract}
Sugarcane is one of the most important crops of the Brazilian agribusiness and this importance justifies the use of techniques that allow the implementation of cultivation systems capable of reducing the variability of soil characteristics and the establishment of efficient agricultural planning. The aim of the present work was the planning and implementation of cultivation systems for sugarcane using geostatistical techniques, in the Pereira Barreto region, SP. An area of 505 ha was mapped using the global positioning system (GPS) and a Digital Elevation Model was elaborated (MDE). Soil samples were collected for 0-0.25 m depth, in each 7 ha, for their chemical attributes and texture characterization. Data were analyzed by descriptive statistics and geostatistics. The determination of the spatial distribution of soil granulometric and chemical attributes allowed the allocation of the studied sugarcane varieties according to soil fertility and clay content. The kriging maps of soil granulometric and chemical attributes brought useful information to the establishment of production environments with different soil and crop managements. The identification of different environments by means of geostatistical techniques allowed the precise planning of the sugarcane cultivation, as well as the adequacy of fertilization practices and the allocation of suitable sugarcane varieties adapted to the conditions imposed by differences in the soil attributes.
\end{abstract}

Key words: soil attributes, specific management zones, kriging

1 Parte da Dissertação de Mestrado do primeiro autor apresentada à UNESP, Jaboticabal, SP

2 FCAV/UNESP, CEP 14870-900, Jaboticabal, SP. Fone: (16) 3209-2672. E-mail: marques@fcav.unesp.br; montana@fcav.unesp.br; agromccc@yahoo.com.br; 3209-2624. E-mail: genertp@fcav.unesp.br

3 FEAGRI/UNICAMP, CP 6011, CEP 13083-970, Campinas, SP. Fone: (19) 3521-1069. E-mail: zigomarms@agr.unicamp.br 


\section{INTRODUÇÃO}

O planejamento da agricultura é uma prática cada vez mais comum entre os médios e grandes produtores rurais. A adoção deste sistema tem, por finalidade, maximizar o rendimento das culturas e, conseqüentemente, os lucros, além de minimizar os custos de produção, visto que esta técnica é baseada na identificação e eliminação das possíveis causas de redução da produtividade (Liu et al., 2006).

De acordo com Pennock (2003), a elaboração do modelo de elevação digital (MDE) permite observar as variações topográficas do terreno. A partir daqui, verificar o padrão e distribuição dos fluxos d'água no terreno e, estabelecer relações entre as formas do terreno e a variabilidade dos atributos do solo. Além disso, Schoorl et al. (2000) afirmam que o uso desses modelos favorece o entendimento dos processos de erosão e deposição de sedimentos e a redistribuição dos solos em função desses processos ao longo de uma série histórica destes eventos. Os MDE fornecem informações complementares das áreas de estudo que dispõem de mapas de solos convencionais, auxiliando na elaboração de zonas de manejo do solo e de culturas, auxiliando no planejamento agrícola (Ziadat, 2005).

Várias são as tecnologias levadas a campo as quais permitem a utilização de um sistema de manejo que considera a variabilidade espacial de fatores ligados à produção das culturas e atributos do solo. Dentre essas tecnologias podese destacar o uso e as aplicações de técnicas geoestatísticas, que se baseia na teoria das variáveis regionalizadas (Goovaerts, 2001). Os mapas de variabilidade espacial dos atributos do solo fornecem informações importantes para o estabelecimento de práticas de manejo adequado do solo e das culturas, visto que o comportamento das plantas tende a acompanhar a distribuição espacial e a variação de nutrientes no solo (Miller et al., 1988).

De acordo com Johnson \& Richard (2005), a importância econômica da cultura da cana-de-açúcar justifica os estudos de variabilidade espacial dos atributos do solo. Com o uso dessas técnicas, há maior captação das variações ocorridas nas áreas cultivadas o que poderá propiciar a implantação de um sistema de cultivo comercial que reduza a variabilidade das características intrínsecas do solo e propicie o estabelecimento do planejamento agrícola adequado. Assim, as áreas com características similares entre si, receberiam um mesmo conjunto de práticas agronômicas e zonas com comportamento diferenciado, ou seja, um conjunto de práticas distintas (Flowers et al., 2005).

A adoção desta tecnologia poderá ser justificada pelo fato de que, atualmente, a grande maioria dos produtores de cana-de-açúcar considera uniforme o solo de cada área de cultivo. Entretanto, cada talhão poderá ter variações consideráveis em seus atributos, como variabilidade do tipo de solo, da produtividade, da declividade e da necessidade de nutrientes, ante o que se propôs, através do presente estudo, o planejamento agrícola e a implantação de um sistema de cultivo de cana-de-açúcar com o auxílio de técnicas geoestatísticas, na região de Pereira Barreto, SP.

\section{MATERIAL E MÉTODOS}

A área de estudo localiza-se no nordeste do Estado de São Paulo, no município de Pereira Barreto, SP (2041' 15” S e $51^{\circ} 03$ ’ 45” W), na província geomorfológica do Planalto Ocidental Paulista. O relevo é predominantemente plano a suave ondulado, com declividades médias variando de 3 a 5\% e altitude oscilando entre 335 a 390 m acima do nível do mar. Nesta região predominam os Latossolos, que se distribuem ao longo da vertente, com curvaturas retilíneas e convexas. Nas posições mais elevadas e planas se encontram o Latossolo Vermelho distrófico textura média, cujo material de origem arenitos da Formação Santo Anastácio, os quais transicionam encosta abaixo, e o Latossolo Vermelho eutroférrico, textura argilosa, originado sobretudo dos produtos da alteração dos basaltos Formação Serra Geral (IPT, 1981).

Segundo a classificação de Köppen, o clima da região é do tipo tropical chuvoso, de bosque com chuvas de verão e estiagem no inverno (Aw), temperaturas variando entre 26,2 e $21^{\circ} \mathrm{C}$ e precipitação média de $1.128 \mathrm{~mm}$, com chuvas concentradas no período de novembro a fevereiro. A área de estudo é, atualmente, cultivada com cana-de-açúcar, que substituiu as pastagens.

Realizou-se o mapeamento de uma área de 505 ha através do equipamento de sistema de posicionamento global (GPS); em seguida, elaborou-se o Modelo de Elevação Digital (MED), por meio do programa Surfer (Golden Software, 1999), que possibilitou a visualização da área (Figura 1). Coletaram-se, por fim, amostras de solo, para análise química e granulométrica, nos pontos de cruzamento de uma malha com intervalos de $350 \mathrm{~m}$ na profundidade de 0-0,25 m a cada 7 ha formando, uma malha contendo 67 pontos. Os ambientes de produção foram estabelecidos com base nos dados de fertilidade (CTC, MO, SB, V\%, pH e $\mathrm{H}+\mathrm{Al}$ ) contando com auxílio do programa Surfer.

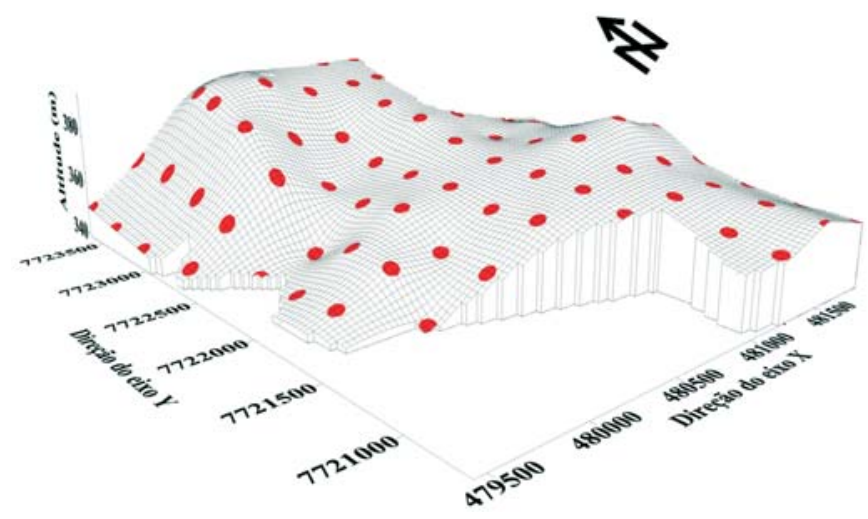

Figura 1. Modelo de Elevação Digital da área estudada e respectivos locais de amostragem $(\bullet)$

Determinaram-se, na caracterização química do solo a acidez ativa ( $\mathrm{pH}$ em $\mathrm{CaCl}_{2}$ ), potenciometricamente, por meio da relação 1:2,5 de solo: em $\mathrm{CaCl}_{2}$ e a acidez potencial $\left(\mathrm{H}^{+}+\mathrm{Al}^{3+}\right)$ segundo Raij et al. (2001). As bases trocáveis cálcio $(\mathrm{Ca})$, magnésio $(\mathrm{Mg})$ e potássio $(\mathrm{K})$, foram extraídas 
utilizando-se o método da resina trocadora de íons, proposta por Raij et al. (2001) e se calcularam, com base nos resultados das análises químicas, os valores da soma de bases (SB), a capacidade de troca catiônica (CTC) e a saturação por bases (V\%). A determinação do conteúdo de matéria orgânica foi realizada segundo metodologia proposta pela EMBRAPA (1997).

A análise granulométrica foi realizada pelo método da pipeta, com uma solução de $\mathrm{NaOH}$ 0,1 N como dispersante químico e agitação mecânica em aparato de baixa rotação, durante 16 h, seguindo-se metodologia da EMBRAPA (1997). A fração argila foi separada por sedimentação, a areia grossa e fina por tamisação e o silte calculado por diferença.

Para adubação de base da cana-de-açúcar utilizou-se a formulação 4-20-20 na forma sólida e para adubação complementar, torta de filtro na forma líquida, de acordo com a necessidade de cada ambiente de produção (Raij et al., 1996). Selecionaram-se, a partir dos ambientes de produção, as variedades de cana-de-açúcar, tolerantes a fertilidade do solo (baixa, média e alta fertilidade), ou seja, as mais exigentes em fertilidade foram plantadas nos ambientes com os atributos mais favoráveis ao seu desenvolvimento. Outro aspecto relevante foi a tolerância das variedades ao stress hídrico avaliado de forma indireta, pelos teores de areia e argila. As variedades utilizadas foram: RB72-454, RB83-5054, RB84-5257, RB85-5453, RB86-7515, SP80-185, SP80-1816, SP80-3480, SP81-3250, SP83-2847, SP83-5070, SP87-365, SP89-1115, SP91-1049, SP91-3011 e IAC87-3396.

Os atributos granulométricos e químicos do solo foram primeiramente analisados por meio da análise estatística descritiva, calculando-se a média, mediana, desvio padrão, variância, coeficiente de variação, coeficiente de assimetria e curtose. A hipótese de normalidade dos dados foi definida pelo teste de Kolmogorov-Smirnov, por meio do programa computacional Sas (Schlotzhaver \& Littell, 1997) enquanto a dependência espacial foi avaliada pelos ajustes de semivariogramas (Vieira et al., 1983), pressupondo a estacionariedade da hipótese intrínseca e pode ser definido como:

$$
\hat{\gamma}(h)=\frac{1}{2 N(h)} \sum_{i=1}^{N(h)}\left[Z\left(x_{i}\right)-Z\left(x_{i}+h\right)\right]^{2}
$$

em que $\mathrm{N}$ (h) é o número de pares experimentais de observações $\mathrm{Z}\left(\mathrm{x}_{\mathrm{i}}\right)$ e $\mathrm{Z}\left(\mathrm{x}_{\mathrm{i}}+\mathrm{h}\right)$ separados por uma distância $\mathrm{h}$. O semivariograma é representado pelo gráfico $\hat{\gamma}(\mathrm{h})$ versus $h$. Do ajuste de um modelo matemático aos valores calculados de $\hat{\gamma}(h)$, são estimados os coeficientes do modelo teórico para o semivariograma (o efeito pepita, $\mathrm{C}_{0}$; patamar, $\mathrm{C}_{0}+\mathrm{C}_{1}$ e o alcance, a). Quanto menor o alcance mais rapidamente se obtém a independência entre amostras uma vez que o alcance é a distância limite da dependência espacial. A variabilidade extrínseca, relativa às práticas de manejo do solo, contribui para a redução do alcance (Isaaks \& Srivastava, 1989).

De acordo com Mcbratney \& Webster (1986) os modelos de semivariogramas esférico, exponencial, linear, linear com patamar e gaussiano são os mais adotados. Os modelos dos semivariogramas foram ajustados por meio do programa $\mathrm{GS}^{+}$ (Robertson, 1998) e posteriormente usados no desenvolvimento de mapas de isolinhas (krigagem). Em caso de dúvida entre mais de um modelo para o mesmo semivariograma, considerou-se o maior valor do coeficiente de correlação obtido pelo método de validação cruzada e menor SQR (soma de quadrados do resíduo). Para elaboração dos mapas de distribuição espacial das variáveis empregou-se o programa Surfer (Golden Software, 1999).

\section{RESULTADOS E DISCUSSÃO}

Os resultados referentes ao teste Kolmogorov-Smirnov para os atributos granulométricos e químicos do solo, indicaram normalidade para as variáveis $\mathrm{pH}, \mathrm{H}+\mathrm{Al}$ e V\% (Tabela 1). Os valores da média e da mediana para todas as variáveis, estão próximos, mostrando distribuições simétricas, com exceção da variável silte. A normalidade dos dados não é uma exigência da geoestatística, é conveniente apenas que a distribuição não apresente caudas muito alongadas, o que poderia comprometer as estimativas da krigagem, as quais são baseadas nos valores médios (Isaaks \& Srivastava, 1989). Mais importante que a normalidade dos dados é a ocorrência do efeito proporcional em que a média e a variância dos dados não sejam constante na área de estudo, evento não observado, ou seja, ocorrência de estacionaridade de segunda

Tabela 1. Estatística descritiva para as variáveis argila, silte, areia, pH, matéria orgânica, acidez potencial, soma de bases, capacidade de troca catiônica e saturação por bases das amostras coletadas nos pontos da malha de amostragem

\begin{tabular}{|c|c|c|c|c|c|c|c|c|c|}
\hline \multirow[b]{2}{*}{$\begin{array}{l}\text { Estatística } \\
\text { Descritiva }\end{array}$} & \multicolumn{3}{|c|}{ Atributos granulométricos } & \multicolumn{6}{|c|}{ Atributos químicos } \\
\hline & Areia & $\begin{array}{l}\text { Silte } \\
\left(\text { q }^{-1-1}\right)\end{array}$ & Argila & $\mathrm{pH}$ & $\underset{\left(g^{\mathrm{kg}}{ }^{-1}\right)}{\mathrm{MO}}$ & $\mathrm{H}+\mathrm{Al}$ & SB & CTC & $\begin{array}{c}V \\
(\%)\end{array}$ \\
\hline Média & 673,10 & 104,30 & 223,43 & 5,05 & 16,95 & 24,00 & 30,46 & 54,46 & 52,58 \\
\hline Mediana & 672,00 & 90,00 & 220,00 & 5,10 & 16,00 & 25,00 & 29,36 & 52,00 & 53,05 \\
\hline Mínimo & 240,00 & 20,00 & 90,00 & 4,50 & 8,00 & 16,00 & 11,60 & 32,00 & 32,25 \\
\hline Máximo & 880,00 & 350,00 & 510,00 & 5,70 & 35,00 & 38,00 & 112,40 & 140,00 & 80,06 \\
\hline $\mathrm{DP}(1)$ & 154,80 & 58,88 & 60,45 & 0,26 & 3,55 & 4,35 & 19,14 & 21,47 & 10,95 \\
\hline $\mathrm{CV}^{(2)}(\%)$ & 22,99 & 56,44 & 27,05 & 5,14 & 20,94 & 18,13 & 62,83 & 39,42 & 20,82 \\
\hline Assimetria & $-1,18$ & 1,80 & 1,06 & $-0,25$ & 1,29 & 0,47 & 2,15 & 1,85 & 0,12 \\
\hline Curtose & 0,62 & 4,18 & 0,28 & $-0,07$ & 2,76 & 0,31 & 6,04 & 4,20 & $-0,30$ \\
\hline$d^{(3)}$ & 0,16 & 0,16 & 0,15 & $0,14^{\text {ns }}$ & 0,18 & $0,13^{\text {ns }}$ & 0,17 & 0,16 & $0,08^{\text {ns }}$ \\
\hline
\end{tabular}

${ }_{1}^{\mathrm{DP}}$ - desvio padrão; ${ }^{2} \mathrm{CV}$ - coeficiente de variação; ${ }^{3} \mathrm{~d}$ - teste de normalidade, nsnão significativo pelo teste de Kolmogorov-Smirnov 
ordem necessária ao uso da geoestatística, a qual pode ser observada pelos patamares bem definidos dos semivariogramas ajustados (Figura 2).

Coeficientes de variação (CV) são adimensionais e permitem a comparação de valores entre diferentes atributos do solo. Valores elevados de CV podem ser considerados os primeiros indicadores da existência de heterogeneidade nos dados. A variação dos atributos do solo, por sua vez, podem ser classificadas de acordo com o coeficiente de variação (CV) conforme destacam Warrick \& Nielsen (1980). A vari-
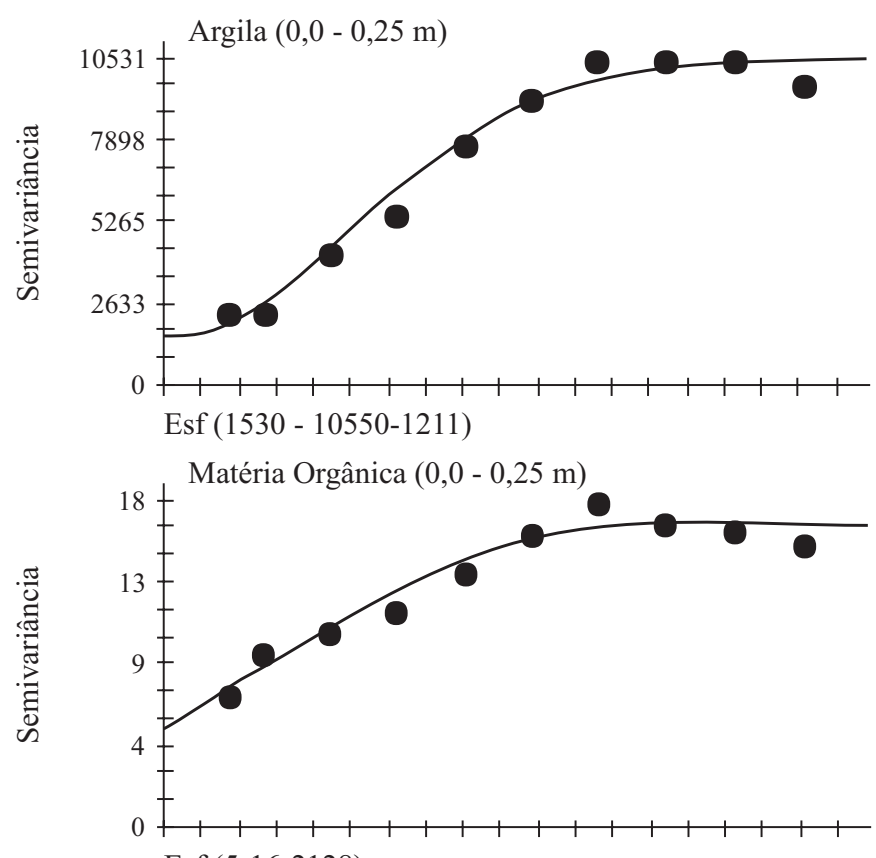

Esf (5-16-2128)
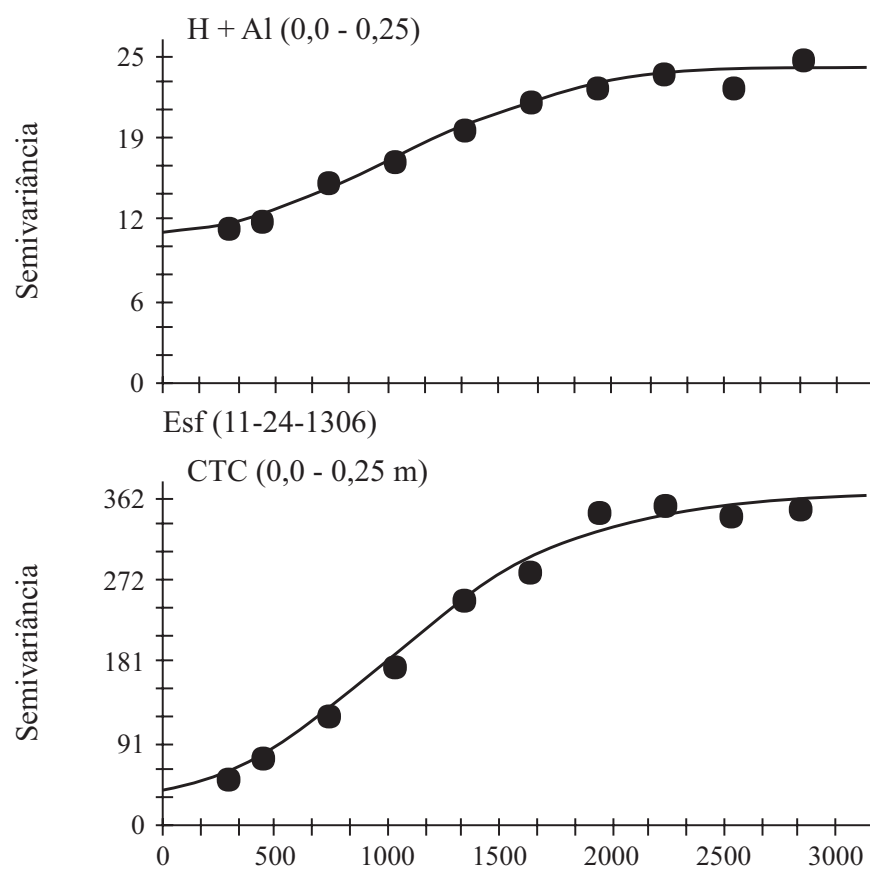

Esf (39-363-1309) ável pH apresentou baixa variabilidade. Para os atributos areia, $\mathrm{MO}, \mathrm{H}+\mathrm{Al}$ e V\%, o CV foi moderado; para as demais variáveis, a variabilidade foi considerada alta (Tabela 1).

De acordo com os resultados da análise geoestatística, todas as variáveis estudadas apresentaram dependência espacial (Figura 2). O coeficiente de determinação do teste de validação cruzada para os atributos areia, silte, argila, $\mathrm{pH}, \mathrm{MO}$, $\mathrm{H}+\mathrm{Al}, \mathrm{SB}, \mathrm{CTC}$ e V\% foram de 61, 57, 59, 65, 61, 58, 60, 64 e $69 \%$, respectivamente. Os dados das variáveis estudadas se ajustaram ao modelo gaussiano, com exceção das variáveis
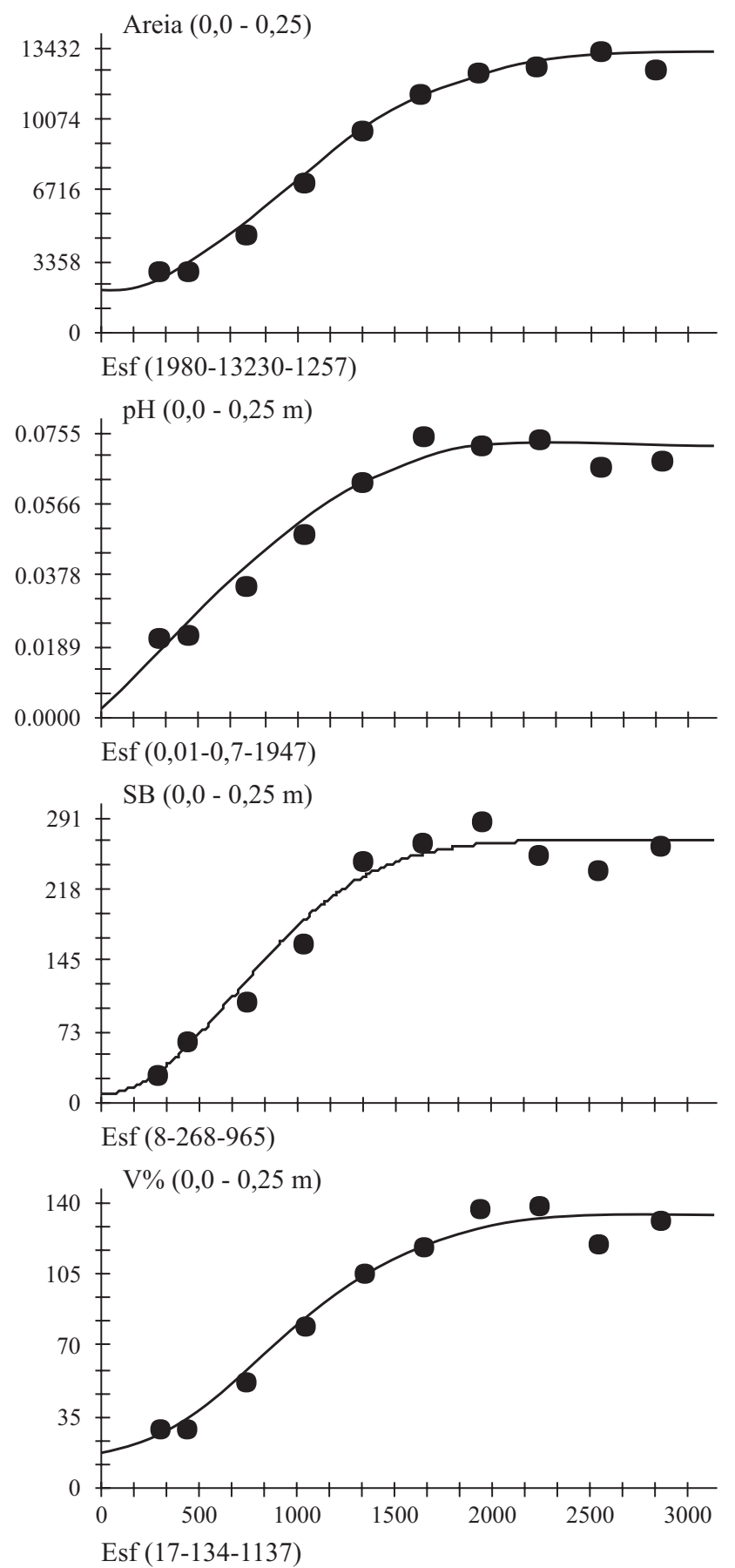

Distância (m)

Figura 2. Semivariogramas das variáveis argila $\left(\mathrm{g} \mathrm{kg}^{-1}\right)$, areia $\left(\mathrm{g} \mathrm{kg}^{-1}\right)$, teor matéria orgânica $\left(\mathrm{g} \mathrm{kg}^{-1}\right)$, soma de bases $\left(\mathrm{mmol}_{\mathrm{C}} \mathrm{dm}^{-3}\right)$, acidez potencial $\left(\mathrm{mmol}_{\mathrm{C}} \mathrm{dm}^{-3}\right)$, capacidade de troca catiônica $\left(\mathrm{mmol}_{\mathrm{C}} \mathrm{dm}^{-3}\right)$ e saturação por bases $(\mathrm{V} \%)$ na profundidade de $0-0,25 \mathrm{~m}$. Esf. e Gaus $\left(\mathrm{C}_{0} ; \mathrm{C}_{0}+\mathrm{C}_{1} ;\right.$ a) $\mathrm{Esf}=$ modelo esférico, Gaus = modelo gaussiano, $\mathrm{C}_{0}=$ efeito pepita; $\mathrm{C}_{0}+\mathrm{C}_{1}=$ patamar; $\mathrm{a}=$ alcance 
pH e MO, que se ajustaram ao modelo esférico. Os atributos granulométricos indicaram valores bem próximos de alcance, ou seja, a argila com valor de $1.211 \mathrm{~m}$ e a areia de $1.257 \mathrm{~m}$. Segundo Souza et al. (2004) o teor de argila e areia dos latossolos são atributos estáveis, ou seja, modificam-se pouco ao longo do tempo por ação do intemperismo, portanto, apresentam baixos valores de CV e valores de alcances próximos. Os atributos químicos mostraram valores de alcance variando de $965 \mathrm{~m}$ (SB) até $2.128 \mathrm{~m}$ (MO).

O mapa de krigagem da fração areia do solo indicou os
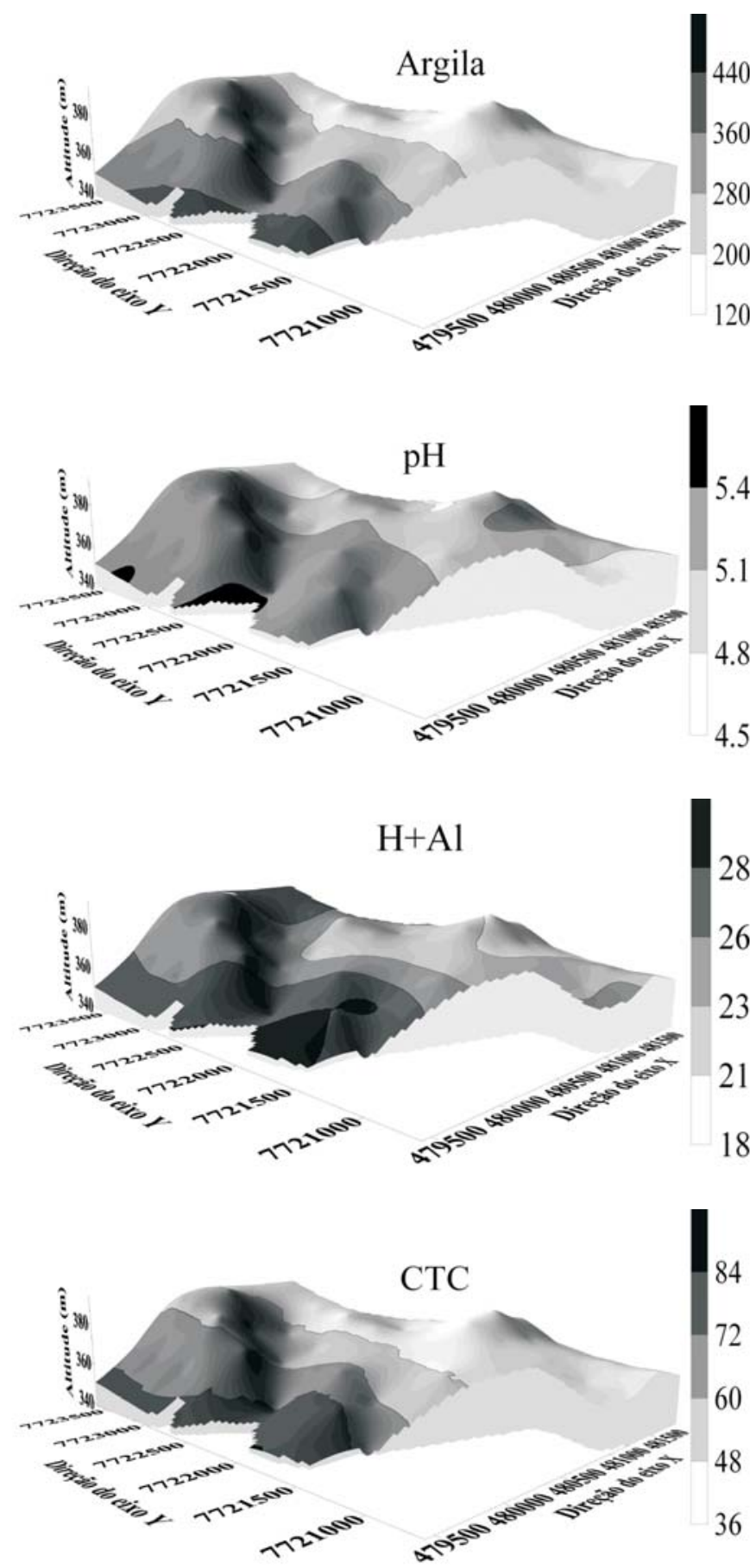

maiores conteúdos nas partes mais altas do terreno, influenciadas pelo material de origem (arenitos da Formação Santo Anastácio), que exibia grandes quantidades de areia fina a média originária de depósitos fluviais (Figura 3). Comportamento contrário pode ser observado em relação ao conteúdo de argila, que se encontram mais concentrado nas partes de menores cotas da área em estudo, fato que se deve, sobremaneira, aos derrames basálticos da Formação Serra Geral que originaram esses solos; resultados semelhantes foram observados por Iqbal et al. (2005).
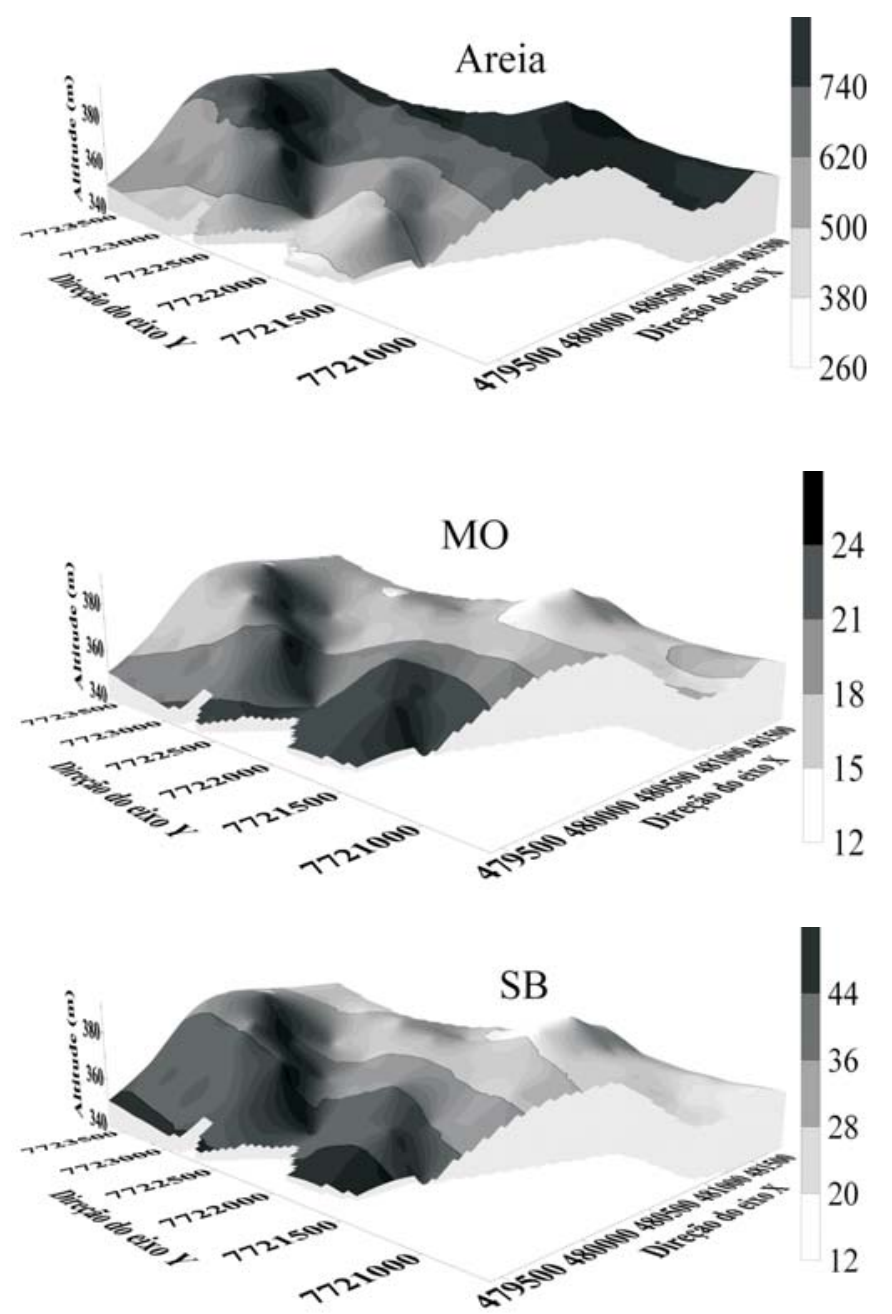

44 36 28 20 12

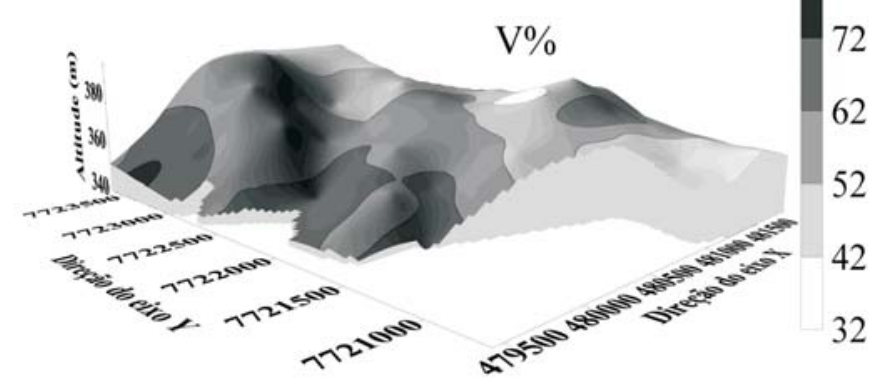

Figura 3. Mapas de krigagem do teor de argila $\left(\mathrm{g} \mathrm{kg}^{-1}\right)$, areia $\left(\mathrm{g} \mathrm{kg}^{-1}\right)$, $\mathrm{pH}$, conteúdo de matéria orgânica $-\mathrm{MO}\left(\mathrm{g} \mathrm{kg}^{-1}\right)$, acidez potencial $\left(\mathrm{mmol}_{\mathrm{C}} \mathrm{dm}^{-3}\right)$, soma de bases $\left(\mathrm{mmol}_{\mathrm{C}} \mathrm{dm}^{-3}\right)$, capacidade de troca catiônica - CTC $\left(\mathrm{mmol}_{\mathrm{C}} \mathrm{dm}^{-3}\right)$ e saturação por bases - V $(\%)$ na profundidade de $0-0,25 \mathrm{~m}$ 
A distribuição das variáveis químicas foi semelhante às variáveis texturais (Figura 3) e os teores de $\mathrm{pH}, \mathrm{MO}, \mathrm{SB}$ e CTC acompanharam o padrão de distribuição da fração argila do solo, com valores mais expressivos nas partes mais baixas do terreno onde o relevo é mais movimentado, função principalmente do material de origem, basáltico, reforçado pelo comportamento do relevo, que influencia sobretudo no movimento anisotrópico da água (vertical e horizontal) (Sommer \& Schlichting, 1997; Anjos et al., 1998). A acidez potencial expressa pelo $\left(\mathrm{H}^{+}+\mathrm{Al}^{+3}\right)$ e a saturação por bases (V\%) apresentam os teores mais elevados nos locais em que se encontram os maiores teores de argila e matéria orgânica, em função do balanço de carga.

As informações das variáveis químicas $\left(\mathrm{SB}, \mathrm{H}^{+}+\mathrm{Al}^{3+}\right.$, CTC, pH e V\%, Figura 3) propiciaram a divisão da área em três ambientes diferenciados para a aplicação da adubação em base. O ambiente 1 abrangeu a maior área, com 259,6 ha, onde foram aplicadas as maiores quantidades de adubo, $620 \mathrm{~kg} \mathrm{ha}^{-1}$. Para a zona 2, cuja área de abrangência corresponde a 155,7 ha, foram aplicados $550 \mathrm{~kg} \mathrm{ha}^{-1}$ de adubo. Já na terceira faixa, que compreende uma área de 89,5 ha, representando a menor faixa, necessitou-se das menores quantidades de adubo, no total de $500 \mathrm{~kg} \mathrm{ha}^{-1}$ da formulação 4-20-20, comportamento que reflete, de maneira clara, o material de origem e o grau de intemperização dos solos (Figura 4). Com o uso dessa ferramenta foi possível maior economia de fertilizantes e, ao mesmo tempo, maior eficiência na aplicação, já que permite maior aproveitamento da aplicação localizada.

Os mapas do conteúdo de matéria orgânica e de areia (Figura 3) forneceram informações significativas para a aplicação da adubação complementar com compostagem de torta de filtro formando, assim, duas zonas: a primeira área, com 285 ha, necessitou de $300 \mathrm{~L} \mathrm{ha}^{-1}$ de torta de filtro, enquanto no segundo ambiente, com 219,8 ha, em função dos teores relativos de matéria orgânica elevados, não houve necessidade da aplicação do complemento (Figura 4).

Estabeleceram-se, com base nos mapas de isolinhas, as práticas de manejo do solo e de culturas. Os mapas de krigagem dos dados texturais (areia e argila) e os atributos químicos (SB, $\mathrm{H}^{+}+\mathrm{Al}^{3+}$, CTC, $\mathrm{pH}$ e V\%) permitiram a implantação das variedades de cana-de-açúcar em função da exigência de água e da fertilidade do solo (Figura 3). Distinguindo três grupos de variedades, A seguir implantaramse, nas áreas com os maiores teores de areia e baixos teores de fertilidade, as variedades RB72-454, RB86-7515, SP832847, RB84-5257, SP80-3480, IAC87-3396, SP83-5070 e SP81-3250 que, segundo Landell et al. (1999) e Raizer \& Vencovsky (1999) são mais resistentes ao stress hídrico e não exigentes ou pouco exigentes em fertilidade; essas variedades ocuparam uma área de 247,5 ha (Figura 5). Maule et al. (2001) destacam que a grande potencialidade de produtividade dessas cultivares é função também de suas características genéticas.

Nas áreas cujos solos apresentam maiores níveis de fertilidade e também maiores teores de argila e, portanto, maior disponibilidade de água, foram alocadas as variedades RB83-5054, SP80-185, RB85-5453 e SP80-1816, as quais,
A.

Mapa de recomendação de adubação de base

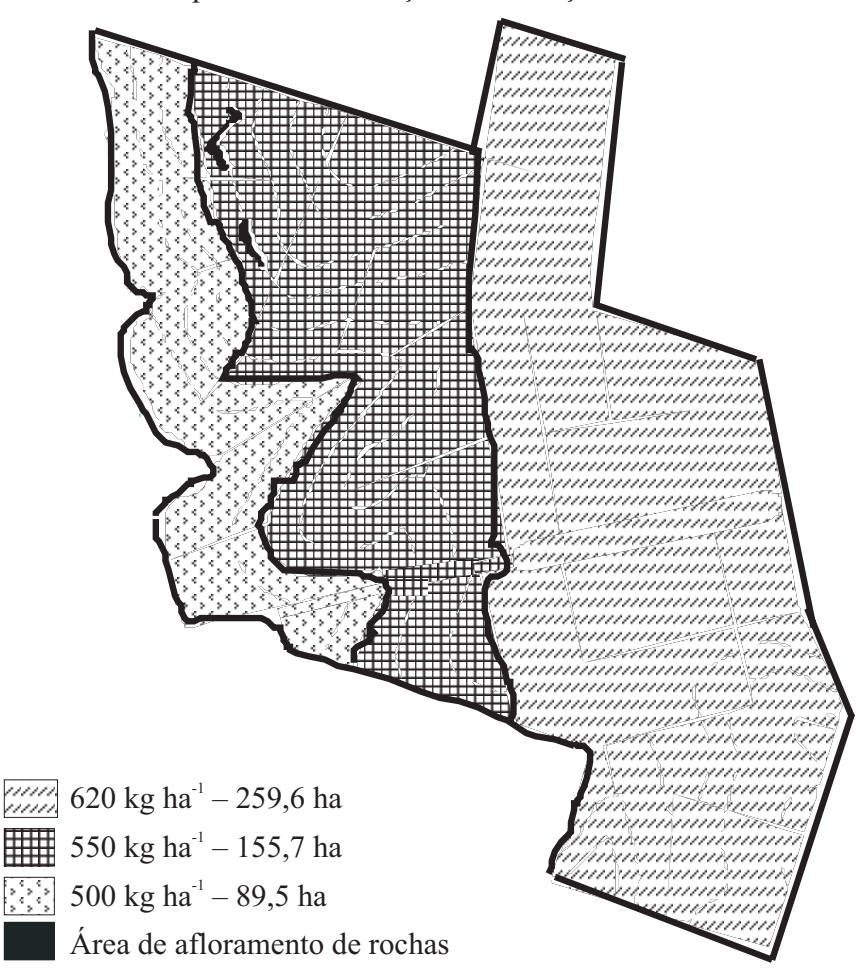

B. Mapa da adubação complementar - torta de filtro

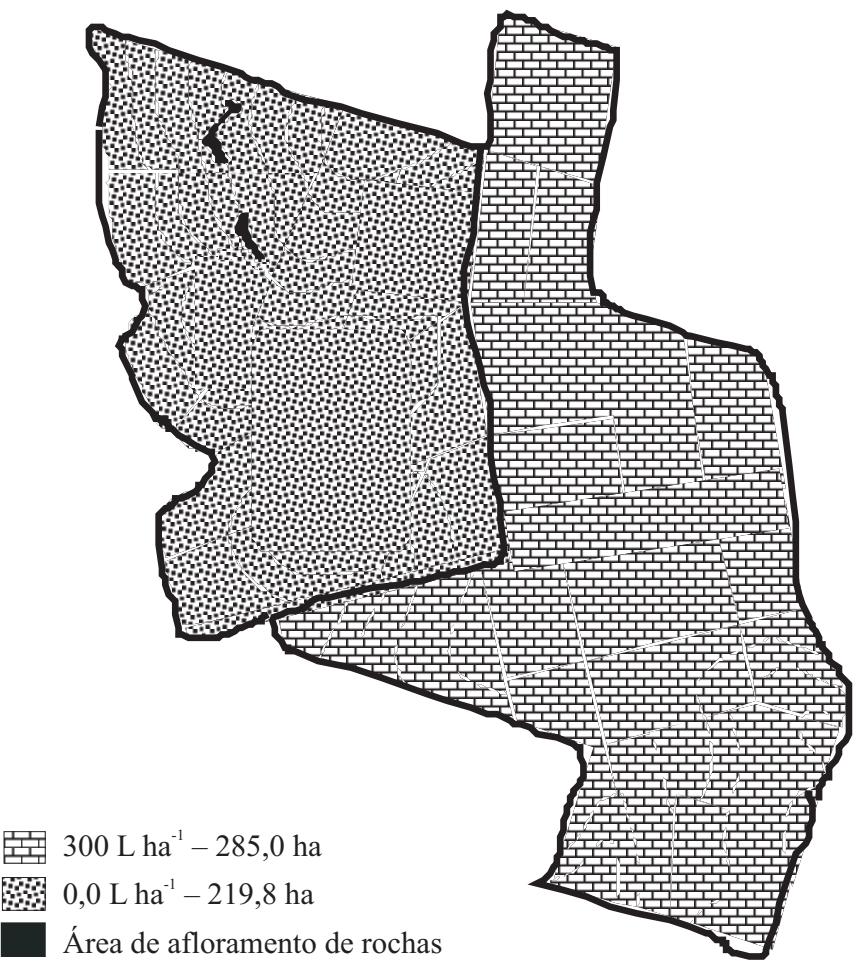

Figura 4. Mapa de recomendação de adubação de base (A) e complementar (B), para uma área de 504,8 ha com cultivo de cana-de-açúcar, no município de Pereira Barreto, SP

segundo Raizer \& Vencovsky (1999) são menos resistentes ao déficit hídrico e exigentes ou muito exigentes em fertilidade do solo, totalizando 135,7 ha. Na faixa intermediária das frações granulométricas e de fertilidade as variedades 
A.

Variedades do grupo I

Variedades do grupo II

Variedades do grupo III

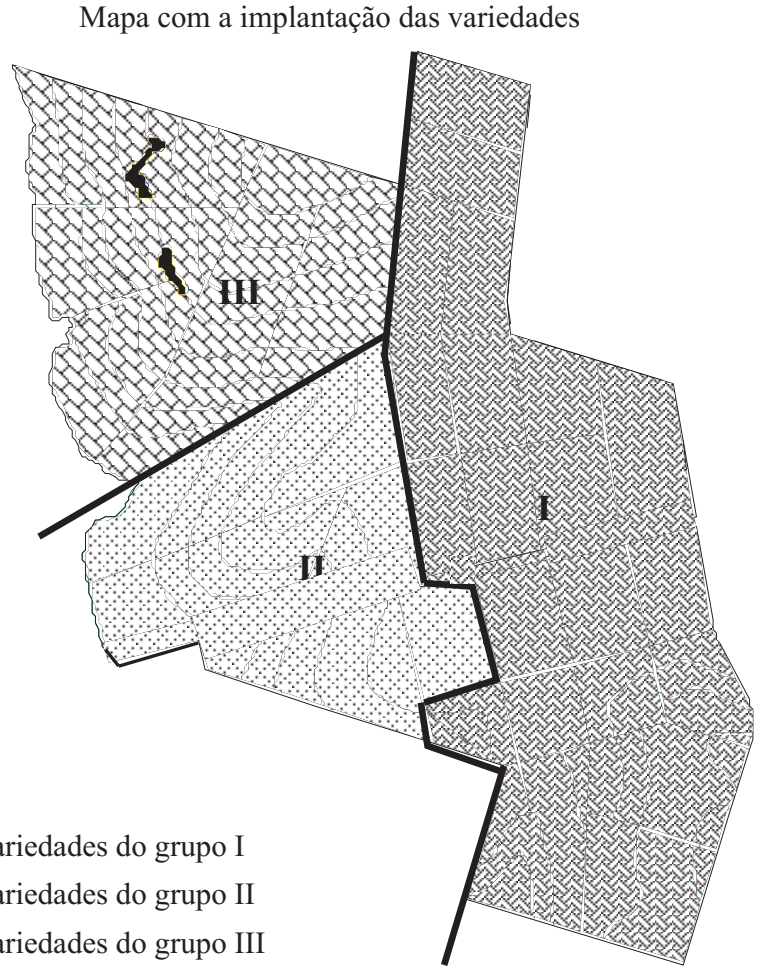

B.

Mapa dos ambientes de produção

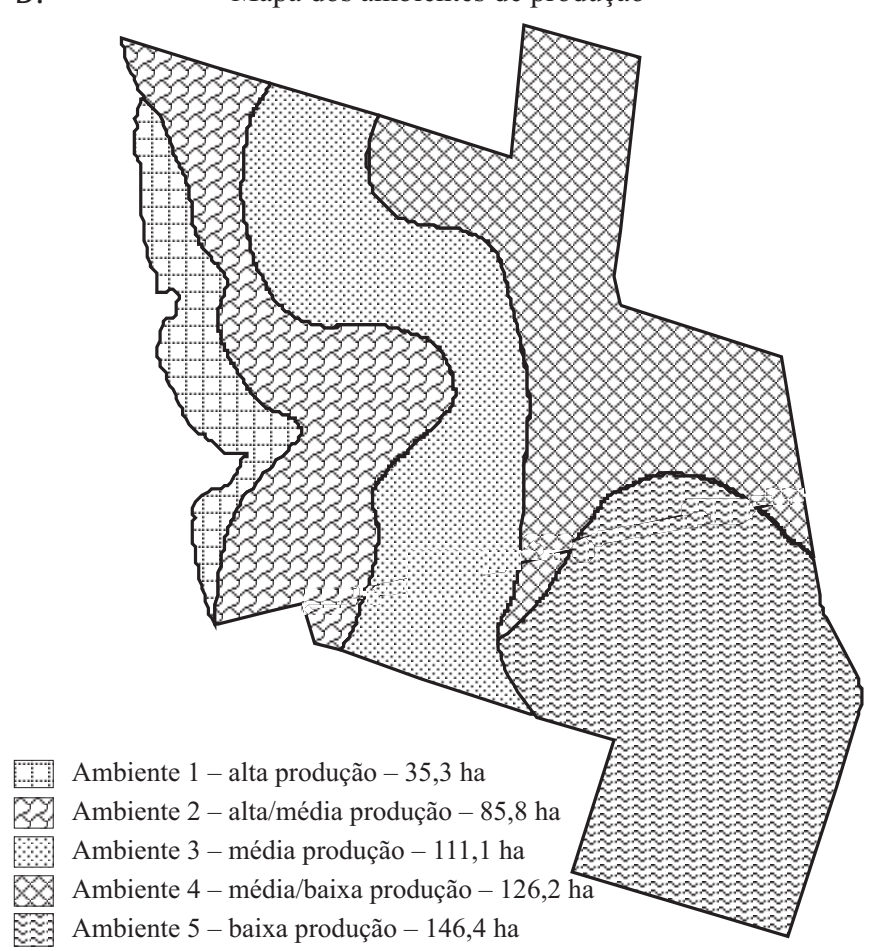

Figura 5. Mapas para a implantação das variedades (A) e ambientes de produção (B) em uma área de 504,8 ha para o cultivo com cana-de-açúcar, no município de Pereira Barreto, SP. I - grupo de variedades não exigentes ou pouco exigentes em fertilidade; II - grupo de variedades com média exigência de fertilidade; III - grupo de variedades exigentes ou muito exigentes em fertilidade

com média exigência de água e em fertilidade do solo (SP91-1049, SP87-365, SP89-1115 e SP91-3011) foram alocadas em uma área de 121,6 ha (Figura 5). A variação do comportamento das cultivares em relação aos ambientes de produção é influenciada também por fatores como solo e cli- ma, que propiciam o desenvolvimento vegetal, expressão do potencial genético de cada cultivar (Dias et al., 1999).

O conjunto dos mapas de krigagem das variáveis granulométrica e química do solo forneceu informações para o estabelecimento dos ambientes de produção, com manejo do solo e de culturas diferenciado (Figura 3). Segundo Farias et al. (2003), a produtividade das culturas varia espacialmente e determinar as causas dessas variações é fundamental para o estabelecimento de zonas específicas de manejo. A partir dessas informações foram estabelecidos cinco ambientes de produção, em que o ambiente 1 foi considerado de altas produções, e abrange uma área de aproximadamente 35,3 ha; o ambiente 2 foi reconhecido como de média a alta produção, com uma área de 85,8 ha, enquanto o ambiente 3 foi acatado como de médias produções, ocupando 111,1 ha. Já o ambiente 4 , de média a baixa produção, cuja área perfaz 126,2 ha e, finalmente, o ambiente 5, foi considerado de baixa produção, com área de 146,4 ha (Figura 5). Resultados similares de distribuição espacial foram encontrados por Farias et al. (2003) em pomares cítricos.

\section{CONCLUSÕES}

1. A distribuição espacial dos atributos granulométricos e químicos do solo permitiu alocação das variedades em estudo de acordo com a fertilidade do solo e teor de argila.

2. O mapa de krigagem dos atributos granulométricos e químicos do solo forneceu informações para o estabelecimento dos ambientes de produção, com manejo do solo e de culturas diferenciado.

3. O uso de técnicas geoestatísticas aplicadas na análise dos atributos dos solos permitiu realizar com precisão o planejamento do cultivo de cana-de-açúcar, a partir da separação de ambientes, adequação de práticas de adubação e alocação de variedades adaptadas às restrições impostas pelos atributos dos solos.

\section{AgRADECIMENTOS}

Os autores agradecem à Usina Santa Adélia, pelo apoio e concessão da área de estudo.

\section{LITERATURA CITADA}

Anjos, L. H.; Fernandes, M. R.; Pereira, M. G.; Franzmeier, D. P. Landscape and pedogenesis of na Oxisol-Inceptisol-Ultisol sequence in Southeastern Brazil. Soil Science Society of America Journal, v.62, n.6, p.1651-1658, 1998.

Dias, F. L. F.; Mazza, J. A.; Matsuoka, S.; Perecin, D.; Maule, R. F. Produtividade de cana-de-açúcar em relação ao clima e solos da Região Noroeste do Estado de São Paulo. Revista Brasileira de Ciência do Solo, v.23, n.3, p.627-634, 1999.

EMBRAPA - Empresa Brasileira de Pesquisa Agropecuária - Centro Nacional de Pesquisa de Solos: Manual de métodos de análise de solo. 2.ed. Rio de Janeiro: Embrapa-CNPS, 1997. 212p. 
Farias, P. R. S.; Nociti, L. A. S.; Barbosa, J. C.; Perecin, D. Agricultura de precisão: mapeamento da produtividade em pomares cítricos usando geoestatística. Revista Brasileira de Fruticultura, v.25, n.2, p.235-241, 2003.

Flowers, M.; Weisz, R.; White, J. G. Yield-based management zones and grid sampling strategies describing soil test and nutrient variability. Agronomy Journal, v.97, n.3, p.968-982, 2005.

Golden Software Inc. (Golden, Estados Unidos). Surfer for windows: realese 7.0, contouring and 3D surface mapping for scientist's engineers user's guide. New York, 1999. 619p.

Goovaerts, P. Geostatistical modeling of uncertainty in soil science. Geoderma, v.103, n.1-2, p.3-36, 2001.

IPT - Instituto de Pesquisas Tecnológicas do Estado de São Paulo. Mapa Geológico do Estado de São Paulo. Escala - 1:500.000. São Paulo: ITP, 1981. v.1, 126p.

Iqbal, J.; Read, J. J.; Thomasson, A. J.; Jenkins, J. N. Relationships between soil-landscape and dryland cotton lint yield. Soil Science Society of America Journal, v.69, n.3, p.872-882, 2005.

Isaaks, E. H.; Srivastava, R. M. An introduction to applied geoestatistics. New York: Oxford University, 1989. 561p.

Johnson, R. M.; Richard, E. P. Sugarcane yield, sugarcane quality, and soil variability in Louisiana. Agronomy Journal, v.97, n.3, p.760-771, 2005.

Landell, M. G. A.; Alvarez, R.; Zimback, L.; Campana, M. P.; Silva, M. A.; Vila Nova, J. C.; Pereira, A.; Perecin, D.; Gallo, P. B.; Martins, A. L. M.; Kanthack, R. A. D.; Figueiredo, P.; Vasconcelos, A.C.M. Avaliação final de clones IAC de cana-deaçúcar da série 1982, em Latossolo Roxo da região de Ribeirão Preto. Bragantia, v.58, n.2, p.269-280, 1999.

Liu, T. L.; Juang, K.W.; Lee, D. Y. Interpolating soil properties using kriging combined with categorical information of soil maps. Soil Science Society of America Journal, v.70, n.4, p.1200-1209, 2006.

Maule, R. F.; Mazza, J. A.; Martha Júnior, G. B. Produtividade agrícola de cultivares de cana-de-açúcar em diferentes solos e épocas de colheita. Scientia Agricola, v.58, n.2, p.295-301, 2001.

McBratney, A. B.; Webster, R. Choosing functions for semi-variograms of soil properties and fitting them to sampling estimates. Journal of Soil Science, Oxford, v.37, n.4, p.617-639, 1986.
Miller, M. P.; Singer, M. J.; Nielsen, D. R. Spatial variability of wheat yield and soil proprieties on complex hills. Soil Science Society of America Journal, v.52, n.4, p.1133-1141, 1988.

Pennock, D. J. Terrain attributes, landform segmentation, and soil redistribution. Soil and Tillage Research, v.69, n.1-2, p.15-26, 2003.

Raij, B. van; Andrade, J. C.; Cantarella, H.; Quaggio, J. A. (eds.). Análise química para avaliação da fertilidade de solos tropicais. Campinas: IAC, 2001. 285p.

Raij, B. van; Cantarella, H.; Quaggio, J. A.; Furlani, A. M. C. (eds.). Recomendações de adubação e calagem para o Estado de São Paulo. 2.ed. Campinas: Instituto Agronômico, 1996. 285p. Boletim Técnico, 100

Raizer, A. J.; Vencovsky, R. Estabilidade fenotípica de novas variedades de cana-de-açúcar para o Estado de São Paulo. Pesquisa Agropecuária Brasileira, v.34, n.12, p.2241-2246, 1999.

Robertson, G. P. GS ${ }^{+}$geostatistics for the environmental sciences: GS+ user's guide. Plainwell: Gamma Design Software, 1998. 152p.

Schlotzhaver, S. D.; Littell, R. C. SAS: system for elementary statistical analysis. 2.ed. Cary: SAS, 1997. 905p.

Schoorl, J. M., Sonneveld, M. P. W., Veldkamp, A. Three-dimensional landscape process modeling: The effect of DEM resolution. Earth Surface Processes Landforms, v.25, n.9, p.10251034, 2000.

Sommer, M.; Schlichting, E. Archetypes of catenas in respect to matter - A concept for structuring and grouping catenas. Geoderma, v.76, n.1-2, p.1-33, 1997.

Souza, Z. M., Marques Júnior, J., Pereira, G.T., Barbieri, D. M. Variabilidade espacial da textura de um Latossolo Vermelho eutroférrico sob cultivo de cana-de-açúcar. Engenharia Agrícola, v.24, n.2, p.309-319, 2004.

Vieira, S. R.; Hatfield, J. L.; Nielsen, D. R.; Biggar, J. W. Geostatistical theory and application to variability of some agronomical properties. Hilgardia, v.51, n.1, p.1-75, 1983.

Warrick, A. W.; Nielsen, D. R. Spatial variability of soil physical properties in the field. In: Hillel, D. (ed.). Applications of soil physics. New York: Academic Press, 1980. p.319-344.

Ziadat, F. M. Analyzing digital terrain attributes to predict soil attributes for a relatively large area. Soil Science Society of America Journal, v.69, n.5, p.1590-1599, 2005. 